Notice
This is the author copy of the paper:
Amaia Aizpurua, Myriam Arrue, Markel Vigo (2015) Prejudices, mem-
ories, expectations and confidence influence experienced accessibility on
the Web. Computers in Human Behavior $51,152-160$. Elsevier
The published paper is available at http://dx.doi.org/10.1016/j.
chb.2015.04.035
$\begin{aligned} & \text { Note that there might be some inconsistencies between this and the above } \\ & \text { publication so use this copy at your own risk. }\end{aligned}$




\title{
Prejudices, memories, expectations and confidence influence experienced accessibility on the Web
}

\author{
Amaia Aizpurua $^{\mathrm{a}, \mathrm{b}}$, Myriam Arrue $^{\mathrm{a}}$, Markel Vigo $^{\mathrm{b}}$ \\ ${ }^{a}$ School of Computer Science, University of the Basque Country, UPV/EHU, \\ Donostia-San Sebastián, Spain \\ ${ }^{b}$ School of Computer Science, University of Manchester, \\ Manchester, United Kingdom
}

\begin{abstract}
Evidence suggests that compliance with accessibility standards does not always guarantee a satisfying user experience on the Web. The literature indicates that addressing the expectations users have about online content and functionalities is crucial to bridge this gap. We examine the role played by subjectiveness, experience and, particularly, expectations on how users experience the accessibility on the Web. To do so, 11 blind participants were enquired through interviews and questionnaires about 12 tasks they completed in four websites. Thematic analysis on the transcriptions reveals that expectations are often built up on previous experiences and preconceived ideas. Particularly, the content which is explicitly labelled as accessible arises the curiosity and creates high expectations about the accessibility of the website. We also find that, in addition to unmet expectations, prejudices on branding issues and the memories evoked by past experiences or emotional bonds does not only affect the way in which users perceive and experience accessibility, but also the overall user experience. Identifying the nature of expectations is key (i) to formalise more exhaustive user testing protocols and (ii) to complement and complete existing accessibility guidelines.
\end{abstract}

Keywords: Behavioral sciences, Blind users, Screen readers, Web, Web accessibility, User Experience

\section{Introduction}

The Web Content Accessibility Guidelines (WCAG) are the internationally recognised standard for Web accessibility (Caldwell et al., 2008; Chisholm et al. 1999). However, evidence suggests that compliance to these accessibility standards does not necessarily guarantee a satisfying user experience on the Web. Studies that corroborate such evidence state that guidelines compliant websites can be inaccessible for specific users in specific situations. The other way around also applies: non-compliant websites do not necessarily have to pose a challenge 
to users. For instance, Petrie et al. (2004) conducted a user study with 51 participants with disabilities, where the authors observed, identified and classified the difficulties that users encountered. They found that $45 \%$ of the observed problems were not related to any violation of WCAG 1.0 checkpoints (Chisholm et al. 1999). The second version of guidelines, namely WCAG 2.0 (Caldwell et al. 2008) was released to address the weaknesses exhibited by the previous versions and to cater for the technological updates that occurred hitherto. Power et al. (2012) conducted an empirical study about the problems identified by 32 screen reader users on the Web. Results revealed that only $50.4 \%$ of the problems encountered by participants were covered by WCAG 2.0 success criteria (henceforth SC). Consistent coverage figures - measured in terms of the percentage of actual problems addressed by guidelines - were reported by Rømen and Svanæs (2012). Among those problems not covered by these SC, the $13.5 \%$ of all user problems are related to unmet expectations in terms of unexpected content (Power et al., 2012).

Even if guidelines are an invaluable starting point for building accessible sites, the above-mentioned findings indicate that there is a need to explore complementary ways of building accessible websites beyond conformance to guidelines. In this regard, we claim that understanding how users experience and perceive Web accessibility is vital to bridge this gap (see Section 2). We expand on this by exploring how subjective dimensions and especially how user expectations influence on the perception and experience of accessibility. Inspired by the work of McCarthy and Wright (2004), who identified that previous experiences, prejudices and branding are the key dimensions that shape user expectations, we analyse how these dimensions influence the perception of blind users about Web accessibility. To do so, we conducted an exploratory study in which 11 blind participants were enquired about the tasks they had to accomplish in four websites (see Section 3). These websites contained different accessibility problems, aesthetic properties and experiential values. The study included semi-structured interviews, user observation and questionnaires that participants filled out.

Our analysis (see Section 4 ) reveals that expectations are built up on previous experiences (either in the physical world or in the Web) and on preconceived ideas. We found that unmet expectations are often related to participants' uncertainty. We also learned that if a Web page is explicitly labelled as accessible and the participant notices it, this does not only spark interest, but also creates expectations that are not always satisfied. As a consequence, if high expectations are not met deception and frustration can be more severe. We also find that memories, past experiences and emotional bonds do also influence the perception of website accessibility. Prejudices towards a brand can influence this perception too. We conclude that all the above does not only affect user perception about the accessibility of a given website, but also the user experience.

The main outcome of this work suggests that users' perceived accessibility, is not only determined by conformance to guidelines, but also by other experiential and more subjective dimensions, as discussed in Section 5 . Specifically, we reach this conclusion by examining the following axes:

- We explore how the dimensions that build user expectations including past 
experiences and preconceptions reflect on the navigation of blind users on the Web.

- We uncover how these dimensions and their consequences determine how blind users perceive the accessibility of a website.

- We explore the association between real life experiences and Web experiences.

- We discuss how a website is actually perceived when it contains an 'accessible' version.

\section{Background}

In recent years efforts on Web accessibility have been devoted to defining guidelines, comparing evaluation methods and metrics or providing tool support for intelligent browsing. The publication of the WCAG guidelines has not only led to the implementation of worldwide policie: $\$$, but also to a wide range of automated evaluation tools ${ }^{2}$, supporting developers building accessible websites. Most efforts have been directed to evaluate and measure Web accessibility according to standards with the aim of improving websites (Lazar et al., 2004). Even if those efforts are necessary in order to advance towards having a better understanding of accessibility problems on the Web, they do not seem to be enough. A website may have an adequate level of accessibility, but still not be sufficient for users. Even if a developer or evaluator considers a website to be accessible, users may have a different perception about its accessibility. A website designed to be compliant to accessibility standards fails if users cannot experience that accessibility. Since compliance to accessibility standards does not necessarily guarantee a satisfying experience, we pose that the actual perception and experience of accessibility barriers have to be examined, beyond guidelines.

Users' perceived accessibility does not always match to that represented in terms of compliance to guidelines. There are several factors that play a key role in this regard: for instance, some users have a repertoire of skills which allow them to overcome barriers by employing workarounds, namely coping tactics or strategies (Vigo and Harper, 2013). In other cases, users do not notice barriers if these do not prevent them from accomplishing their tasks.

Nevertheless, individuals find the Web a mean, not only for working or achieving informational goals, but also for different purposes like communication, entertainment, social networking or contributing to building the Web, to name a few. In order to establish the scope of this study, and suggest its generalisability to similar website types we use the classification proposed by De Marsico and Levialdi (2004). They proposed a taxonomy which establishes different categories of websites based on a three-dimensional space, derived from

\footnotetext{
${ }^{1}$ Web Accessibility Policy Resources: http://www.w3.org/WAI/policy-res

${ }^{2} \mathrm{~A}$ complete list of Web accessibility evaluation tools: http://WwW.w3.org/WAI/ER/tools/ complete
} 
Aristotle's rhetorical triangle (ethos, logos, pathos). The three axes of the taxonomy represent different communication features of websites: 1. personal/social, which refers to the different target audiences; 2. site/info is the type of information provided by the website; 3 . communication style refers to the effort made by the designer to address the users' affective sphere.

Considering the wide range of websites that exist and thus, the variety of possibilities offered by the Web, non-instrumental information merits attention too. The ISO 9241-11 (1998) standard supports this claim by establishing that subjective qualities such as learnability can also contribute to the usability of a product. Beyond traditional evaluation techniques in Human-Computer Interaction, which have emphasised more on the instrumental aspects that characterise user interaction (such as error rates and task completion times), the User Experience (UX) is intended to provide a more holistic approach to understand how users experience the interaction. With the aim of bridging the HCI and UX research, Bargas-Avila and Hornbæk (2011) conducted a systematic survey on how previous UX studies address issues like situation, context of use, dimensions, when is UX assessed and how UX data is collected. Since UX takes into account the user interaction from a broader perspective, it brings a wider range of possibilities to our research context by considering subjective aspects related to users' perceptions and expectations.

Little attention has been paid to how blind users experience the accessibility barriers on websites. Generally, as blind users are not specially trained on Web accessibility, their perception of Web accessibility can be very subjective and may not match to that of experts. The initial findings of Aizpurua et al. (2013) indicate that expectations play an important role when it comes to how blind users perceive accessibility barriers. This perception is not only about the website and its accessibility, but about what they expect from their engagement with a website. Also, users may assess the accessibility of website based on UX qualities, which at first sight might have little to do with the website's actual accessibility. Our research builds upon this work and based on a more in-depth analysis, adds new evidence to the relatively unexplored research corpus about the intersection of user experience and Web accessibility.

\section{Method}

The unexplored and subjective nature of the experienced accessibility calls for a preliminary qualitative approach (Adams et al. 2008) that will inform subsequent stages of our research. An exploratory study was conducted to collect the data that was analysed using qualitative research methods. The following sections describe our methodological approach in order to uncover the interplay between experienced Web accessibility and other experiential and subjective aspects.

\subsection{Participants}

Eleven legally blind participants - four female participants-, who were representative of the user group being studied (Sears and Hanson, 2011), were re- 
Table 1: Demographic data and characterisation of expertise on the Web

\begin{tabular}{ccccccc}
\hline Id & Gender & Age & $\begin{array}{c}\text { Familiarity } \\
\text { with the Web }\end{array}$ & $\begin{array}{c}\text { Frequency of } \\
\text { Web use }\end{array}$ & $\begin{array}{c}\text { Self-reported } \\
\text { expertise }\end{array}$ & $\begin{array}{c}\text { Observed } \\
\text { expertise }\end{array}$ \\
\hline P01 & F & 29 & $>7$ years & daily & advanced & expert \\
P02 & F & 29 & $>7$ years & daily & advanced & expert \\
P03 & M & 39 & $>7$ years & daily & advanced & advanced \\
P04 & M & 54 & 4-6 years & daily & intermediate & intermediate \\
P05 & M & 43 & 1-3 years & weekly & beginner & intermediate \\
P06 & M & 21 & 1-3 years & weekly & beginner & beginner \\
P07 & M & 64 & $>7$ years & daily & intermediate & advanced \\
P08 & M & 58 & $>7$ years & daily & intermediate & intermediate \\
P09 & F & 54 & 4-6 years & daily & intermediate & advanced \\
P10 & M & 64 & 4-6 years & daily & beginner & intermediate \\
P11 & F & 42 & $>7$ years & weekly & intermediate & beginner \\
\hline
\end{tabular}

cruited in partnership with the National Organization of Spanish Blind People $(\mathrm{ONCE})^{3}$. The median age of participants was 43 years, with a range of 21-64 years. Table 1 shows demographic data as well as the characterisation of user expertise in two ways: self-reported and observed skills (columns 6 and 7 respectively). The former was collected by asking participants to rate their Web expertise on a four-item scale: expert, advanced, intermediate and beginner. In order to avoid the bias of self-judgements we also assessed Web expertise from the perspective of an external observer. This last indicator was computed using observations of the first author and the reports of the instructor, who trained the participants for improving their computer skills at the local delegation of ONCE. Specifically, the first author paid attention to the strategies employed and the confidence shown when carrying out the tasks. As shown in Table 1 . self-rated and observed skills did not always match. This is in line with the conclusions of a study (van der Geest et al., 2014), which shows that self-rated Internet competence of visually impaired users is not always related to their actual performance on common Internet tasks.

\subsection{Apparatus}

All participants were legally blind and utilised screen readers to navigate on the Web: ten participants were Jaws users (version 10, except P01 and P03 who used version 12) on Internet Explorer/Windows (XP and and Win 7), while just one participant (P02) was a VoiceOver user on Safari over MacOS. The first three participants were observed in the research facility of the HCI laboratory at the School of Computer Science, and participants brought their own laptop. Remaining sessions took place in a room at the ONCE delegation in DonostiaSan Sebastián, where the remaining eight participants used the same laptop and keyboard, which were provided by the ONCE.

\footnotetext{
$\sqrt[3]{\text { http://www. once.es/new/otras-webs/english }}$
} 


\subsection{Stimuli}

In order to let the subjective dimensions emerge, we recruited local participants and selected websites of restaurants that are popular and well-known in the area where they lived. We focused on only one type of website, so that we could establish analogous tasks across different stimuli, and also to minimise confounding factors related to the type of stimuli. According to De Marsico and Levialdi (2004)'s taxonomy, the restaurant websites we used fall onto the categories of commercial sites, are targeted to a general audience and exhibit an informative-seductive communication style.

When we sampled the websites, the main selection criteria was the accessibility level of the websites, as we wanted to study how users experienced websites with different accessibility issues. Unfortunately we did not find any local restaurant website that was fully compliant with WCAG 2.0 level AA. Also, it must be noted that some websites were discarded due to the severe accessibility problems they contained in the homepage. Another selection criteria was about branding issues, W1 and W3 represent internationally well-known restaurants with an innovative character (see Figure 1). Their culinary style is based on creativity, investigation and experimentation. Their 'author's cuisine' has been recognised worldwide by the award of Michelin stars. In contrast, the other two restaurants (W2 and W4), even if they are quite popular in Donostia-San Sebastián, their style is based on traditional Basque cuisine. As it can be observed in Figure 1 the visual design of the websites is aligned with the style of the restaurant: the internationally renowned restaurants (those having an innovative and avant-garde style: W1 and W3) use a reduced colour palette, have had their typographies designed and contain high-quality close-up pictures. On the other hand, the design of the traditional restaurants is less ambitious.

These websites had been chosen by following this selection process: we first listed 25 websites of local restaurants; then we evaluated their homepages, using four automated Web accessibility evaluation tools: AChecker (Gay and Li, 2010), EvalAccess (Abascal et al., 2004), TAW ${ }^{4}$ and WAVE ${ }^{5}$ Based on the automated tests of the aforementioned tools we classified websites in four groups: very accessible, accessible, not so accessible, poorly accessible. Within each group, we narrowed down our selection by evaluating again the homepage and two more Web pages using the WAQM metric Vigo et al. (2007). Then we merged the four groups into two (highly accessible and poorly accessible). Within each group, we classified websites based on branding issues, considering the style of the restaurant: traditional and innovative. After that, we carried out an expert evaluation where the Barrier Walkthrough (BW) method (Brajnik, 2006) was applied. Based on the results, we finally selected the two most and least accessible websites for each type of restaurant (traditional and innovative). Once we had the four websites, we evaluated them according to the WCAG 2.0 AA conformance level. Both, VoiceOver and Jaws screen readers

\footnotetext{
${ }^{4}$ http://www.tawdis.net

5 http://wave.webaim.org/
} 
were used during the manual Web accessibility evaluations performed.

In general, W1 and W2 present higher accessibility levels than W3 and W4. Their homepages satisfy $73 \%, 69 \%, 52 \%$ and $36 \%$ of SC for the AA level of WCAG 2.0 respectively. It must be noted that $\mathrm{W} 1$ is the accessible version of the restaurant's website (although we did not tell participants about that detail). The most severe accessibility issue of $\mathrm{W} 3$ is that the navigation menu consists of seven images, all with the same 'alt' text, which was the word image. In $\mathrm{W} 4$, the main accessibility problem is related to Flash based dynamic content that cannot be accessed by the screen reader.

\subsection{Procedure}

Each session was conducted with one participant at a time. Once the participants were informed about the objectives of the study and the procedure of the session, they signed the consent form. In order to obtain non-biased answers, and make participants feel free to respond as honestly as possible, we highlighted that we had no relationship nor conflict of interest with the websites. We also made it clear that there were no right or wrong answers by emphasising that we were interested in their insights and personal opinions. We explained participants that they were playing the role of testers rather than being the tested subjects. Then, we collected demographic data and enquired participants about their expertise and familiarity with information technology. After that, participants were interviewed about their previous experiences and expectations regarding restaurant websites. Once the interview had finished, they could start to navigate the first website. After the navigation with each website, by means of questionnaires and semi-structured interviews, we collected information about their browsing experience. This information was diverse and encompassed their perceptions about website aspects, the problems they encountered, the accessibility barriers found and the affective considerations evoked by the websites. Each session, was video and audio recorded to enable subsequent analysis. At the end of the session each participant was rewarded with a USB memory stick for taking part.

\subsection{Tasks}

Each participant was asked to try to complete three tasks on each website. These tasks were the same for each website: 1 . freely navigate on the website, in order to become familiar with it; 2 . find information about the gastronomic offer; 3 . find the means offered by the website to make a booking. We prevented a learning effect from happening because each website structured its content in a different manner. However, in order to minimise order effects, the task order was counterbalanced. The idea was to let the users explore the website through tasks which were related to the typical expectations regarding restaurant websites. Hence, the tasks were deliberately not very specific and tried to trigger real world situations, where users have an informational goal in mind but explicit directions to accomplish are absent. Participants were told that the time estimated for

each task was between 5-10 minutes but we highlighted that they could spend 


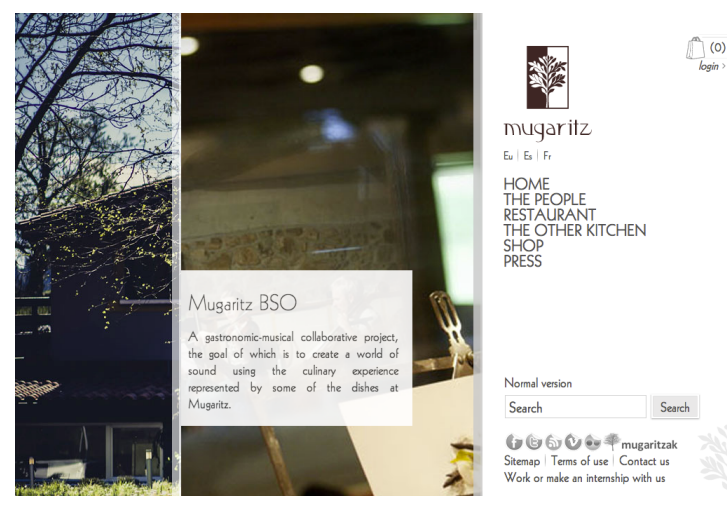

(a) W1

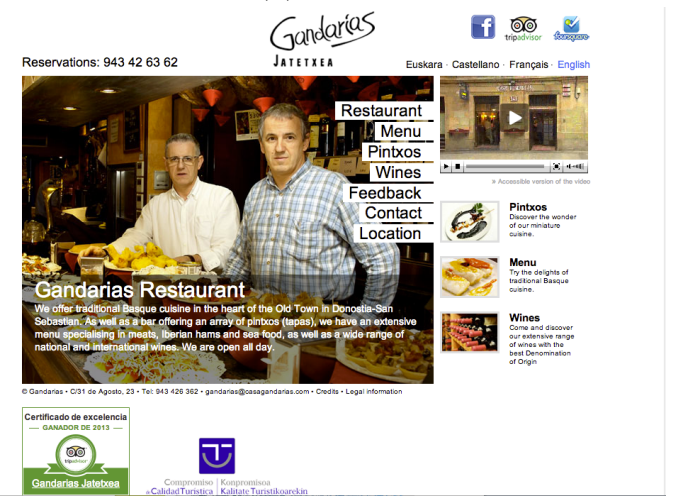

(b) W2

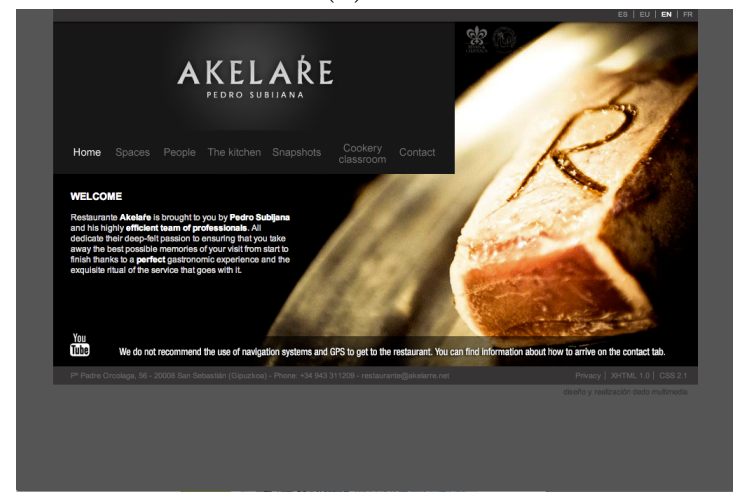

(c) W3

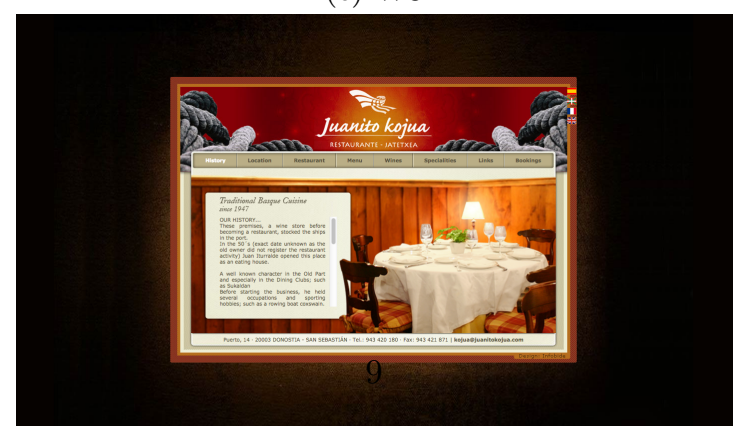

(d) W4

Figure 1: Screenshots of websites. 
longer time if they wanted, or even shorter time if they finished or they had lost their interest.

\subsection{Data Analysis}

We followed the six phases of the thematic analysis (Braun and Clarke, 2006): 1. Familiarising with the data; 2. Generating initial codes; 3. Searching for themes; 4. Reviewing themes; 5. Defining and naming themes; 6. Producing the report. We performed an open coding phase in order to identify the emerging themes in the data. Transcripts were printed on paper and by using highlighters of different colours we identified potential themes. In order to review and validate the identified themes, we analysed all transcripts again using NVivo 10, a qualitative data analysis software. To ensure the reliability of the coding scheme, we asked an external coder to review the transcripts and use the initial coding scheme to code the data. We computed Cohen's Kappa statistic to measure the average level of agreement between both coders and a coefficient of 0.86 was obtained, which indicates a substantial level of agreement. Even if the agreement was quite high, the results were shared and the discrepancies were discussed and resolved. This exercise led to some changes in terms of adding and deleting some instances on the final coding scheme.

\section{Results}

This section presents the themes and sub-themes found with regard to the experiential aspects that emerged from the interaction of participants with the websites 6

\subsection{General Expectations (10)}

We define a expectation as anything expected by the participant, no matter if it is met or not. That is, expectations are any assumption that may or may not be realistic. We learned that the general expectations participants had with regard to the restaurant websites, were built up on to previous experiences: either real (physically at restaurants) or on the Web (with similar websites). Not surprisingly, most participants expected to find information about the gastronomic offer, prices, contact, location, regular timetable or bookings.

Experiences at restaurants. Participants relied on past experiences at restaurants to express what they expected from these websites. Beyond specific content, three participants wanted restaurant websites to provide them with enough and useful information to control the situation when they were physically at the restaurant. As a consequence of having experienced uncomfortable situations in the past, they reported that they usually try to avoid these situations by visiting the website before going to the restaurant. This provides them with

\footnotetext{
${ }^{6}$ The number between parentheses indicates the number of users who reported each subtheme.
} 
useful information to figure out what they can find in the restaurant, and also increases their sense of autonomy and security. This strategy is a way to take precaution to avoid potential uncomfortable situations, and thus, it can facilitate the real experience at the restaurant: "There are many times that you have such a long menu, which is a pain, because the waiters don't even ask you later. They say 'we have this, this and this', when the menu is about 30 dishes! ... but they decide for you what you don't want... Of course, if someone has to read the menu you can spend 15 minutes there reading the menu. I understand that. Well, in order to avoid this I check the menu on the Web" (P02).

Experiences on the Web. In addition to real experiences related to restaurants, past Web experiences with other restaurant websites may also contribute to the creation of expectations. For example, the two participants who habitually visited restaurant websites did not expect them to be accessible as they often came up with many inaccessible websites: "They are very inaccessible, I tell you! Horrible, they are horrible, besides many of them have Flash, and now with HTML5 Flash is not needed ... Online reservations are oof! . . a nightmare ... a nightmare and you end up calling them [on the phone]" (P02).

\subsection{Website Specific Expectations}

General expectations refer to any restaurant website, while specific expectations are related to what participants expected from a particular website. In comparison to general expectations, these are more related to the aspects of a particular website such as content and functionality. While participants navigated a website, the expectations they had with regard to its content or functionality were not always met.

\subsubsection{Content-Related Expectations}

Content found where not expected (4). Encountering content in unexpected locations was not a major problem provided that the content was a relevant piece of information to complete the given task. Some even seem to be surprised of having found it unexpectedly: e.g. the telephone number was very handy at the beginning of the homepage in W2 "We already have a task done! [Laughs]" (P09-W2). However, if they found something they did not want it did not match their mental model participants got confused: "Yes, before the first link, or the ... well, there was 'History', the link was 'Restaurant', well I don't know what it does there" (P03-W4).

Content not found where expected (10). Sometimes participants did not find the content they wanted on a website. Either because of difficulties with the website, the assistive technology, because their search strategy was not effective or because the content they were looking for was not in the website. The majority of the comments from participants were related to information about the given tasks, especially about the gastronomic offer: "The menu was what I wanted to see...the menu and the prices" (P04-W1) and "It does not have no menu nor anything. Because if you go to a website I could not care less about the history, what I want to know is what to eat" (P06-W3). 
Content not found as expected (11). This happened when the content was in a different form than expected by the participant. For instance, P09 thought that the menu would come in a document such as a PDF, or a list of dishes on the same page. However, in the W2 website, information about the gastronomic offer was conveyed through links. In fact, participants had to navigate through different pages that were located at different depths in order to get the information. "The menu, I expected to see a document all together ... and there was no menu as such, it was like a bunch of links" (P09-W2).

Not accessed content (4). Expectations sometimes go beyond the content that participants visited. Sometimes only by the link text, they made assumptions about the content beyond the link, even without exploring the Web page. For instance, one participant imagined that the video on the W3 website was a welcoming video: "There was also a welcoming written text, with an attachment or a link to Youtube, well I understand it's for welcoming, because it was next to the written text" (P03-W3).

Content labelled as 'accessible' (5). It was surprising to observe how the 'accessible' keyword aroused the interest of the participants. It can also generate expectations regarding the content to which it refers. There was a video on the W2 homepage, which had a link next to it that said 'accessible version of the video'. None of the participants played the video neither visited the link of the accessible version of the video but later on the interview four participants mentioned the detail of the accessible video: "I wanted to look at it because it said 'accessible video' ... I forgot to check it out but I wanted to look at it, huh? For me when they put accessible makes me want to try it, right? Then, sometimes you say 'to hell with this accessibility!' Isn't that so? But anyhow, it denotes a level of interest; the designer of the Web may have done it thinking about something or someone ... Well, that is appreciated" (P10-W2).

In addition, as previously mentioned, W1 website was in this case the accessible version of the website. So, it contained a link with the 'normal version' text. As soon as P09 realised that she was navigating on the accessible version of the website she said: "Wow! ... finding an adapted version, even if we say it does not influence our mood, we are already applauding!" (P09-W1). Nevertheless, she did not experienced W1 to be accessible, and after the navigation she said: "As I was browsing I was feeling as if I lacked resources, because I figured that if the version was accessible, it'd be for some reason, and if I wasn't finding information, I was thinking: others [referring to participants] would have found it, because it's accessible, and I look silly because I'm not able to find the information" (P09-W1). In addition, she reported that it is frustrating when something is claimed to be accessible apparently, but finally it turns out that is not: "that bothers me very much ...it's bothering when a company claims to be as such ... then you get disappointed ... it feels like being cheated" (P09-W1).

Beyond the specific case of $\mathrm{W} 1$, we observed different positions and opinions with regard to the accessible or the normal version of a website. Some expert participants said that they usually access the normal version of the websites, 
even when accessing it for the first time. "I always try the other [referring to the "normal" version], but because I want to and I am weird" (P02). In contrast, most of the other participants (non-experts) stated that they usually visit the accessible one if it was available, mainly because they expected to be the most convenient or easiest way. "of course I will always go to the accessible one, I always hit the button to make it more accessible for me ... I don't know in what way 'accessibilizes' it for me, because I do not know much about computers" (P05).

P07 reported that with one particular online newspaper he used to access the text only version, until he realised that it was less complete than the original one. This stopped him from visiting alternative versions of websites and hitherto he sticks to original versions. Another participant said that he usually visits the accessible version of the website, especially if he is browsing in an operative mode, rather than in a more exploratory mode: "yes I go there [to the alternative version], especially if you're in a working attitude, I mean that if you're going to do something in particular" (P10).

We observed two different positions on alternative website versions. Some participants thought that it is not a good approach, and would prefer the website to be accessible without the need of having an alternate version: "I always try the other, and I do not find that page [the accessible version] accessible ...I don't want to be separated ... aren't we going towards the integration?" (P02). In contrast, others feel good about having an accessible version, as long as it is conveniently handy. "I don't go looking for it ... if I knew that all the websites have an accessible feature, I assure you that is the first place I would go ..." (P08).

\subsubsection{Functionality-Related Expectations}

In addition to the content, expectations are also related to the functionality of the website. In this context functionality refers to processes and achievable outcomes.

Expected functionality not present (10). This happened when a website did not provide participants with the expected functionality. For example, in W4 a participant expected that by activating a button he could make a booking: " $I$ wanted to press the button and the reserve by email would show up" (P05-W4).

Functionality does not work as expected (9). This refer to situations where website elements do not work as expected by participants. For instance, links that unexpectedly opened a new window or a new application misled the participants: "I don't know where it has taken me to, to a very strange place, and then you restored it. Well, that could be also as a sign of something that... I cannot tell because I don't know where it took me ... but I was thinking 'well, let's see if it takes me to a section for bookings', that's why I entered, but it didn't happen" (P10-W3). 


\subsection{Preconceptions (9)}

Participants' preconceived ideas regarding the restaurant represented by the website (e.g. branding issues) also affected expectations on the website which can later impact on their perception of the accessibility of the website. Some participants were bothered - and even swore - by the experienced lack of accessibility of the W3 website. The anger was fuelled by the fact that W3 is an internationally well-known restaurant: "If it's so luxurious, they should bother to put it right but they don't care, because as they have lots of money, they would say 'people who know me they know where I am'... Restaurants will say: 'ahh I'm already rich, I don't care if they [referring to blind customers] don't come" (P06-W3).

Surprisingly, two of the restaurants of the websites (W1 and W3) used in our study, had previously carried out initiatives to promote accessibility and get closer to people with visual impairments: the accessible pintx $\oint^{7}$ initiative, and offering the menu in Braille, respectively. In the case of the W1 restaurant, the pintxo was specially created for blind people with the aim that they could enjoy this type of 'social snack', which is very traditional in the area where participants lived. The objective of the initiative was to raise awareness on the difficulties that blind people have to face in their daily routine. Our findings suggest that being aware of these initiatives carried out by restaurants in order to promote accessibility, can influence expectations regarding the accessibility of the website. "Surprised, pleasantly surprised, I did not expect... I knew that the restaurant had done some pro-accessibility things, not on the Web but in general. And that they have been doing some things with the ONCE and such, but I did not think ... yes the accessible pintxo, silly things in my opinion but ... well, maybe that served them to realise that an accessible site is needed" (P02W1). P09 knew that two restaurants had their menus in Braille. Consequently she assumed that their respective websites would be accessible: "maybe you're not going to eat at the Arzak or Akelarre restaurant [two well-known upmarket local restaurants, the latter is the restaurant of W3], but I guess their website is accessible because they have the menu in Braille ... even if it's never updated!" (P09).

\subsection{Evoked Memories (6)}

The participants' memories from their past experiences emerged while carrying out their tasks. These memories were triggered by the recall of the experience of having been at that restaurant, or by content of the website that reminded participants of an emotional bond to someone or something: "I was happy, because apart from having been at Mugaritz [the restaurant corresponding to W1], Mugaritz is located in an area to which I have much affection" (P05-W1). P09 remembered that time when she went to the W4 restaurant with her father. She remembered fondly as an endearing and satisfying experience and expected

${ }^{7}$ A pintxo is the Basque equivalent of a Spanish tapa: a miniature dish you can eat while standing up by the bar. 
the Web experience corresponded to the real one: "I felt happy because I like playing with the pages, and because I was remembering when I had been in [the restaurant of W4] with my father... I've been once or twice. In both cases I had dinner with very few people in the dinning room ... A little disappointed for that reason, because for such a restaurant of quality, and especially for the intimate and satisfying eating experience I've had, nevertheless it's like the website did not match up to my experience" (P09).

P10 commented that he recalled that his parents used to go to have dinner at the restaurant of W4 on the festivities of Donostia-San Sebastián: "I also have remembered that my father and my mother used to go there on the festivities. They used to come to Donostia and then go to this restaurant to have dinner" (P10-W4). Checking the wine list on W2, P10 noticed that there was a selection of wines from the Canary Islands, which caught his attention. He said that he wanted to see if there was a special wine called Malvasia: "I have seen the types of wines it reminded my of the Canary Islands, because there is a typical wine that is the Malvasia ...I entered Canary Islands link" (P10-W2).

\subsection{Uncertainty}

\subsubsection{Confusion Generated by the Website (11)}

We observed that participants' non-met expectations are often related to certain level of uncertainty and confusion. It is not only about expectations that have not been met, but the associated feeling of uncertainty about why they were not fulfilled. For instance, most participants expected to find a menu on the websites. Even if W1 contained information about gastronomic offer, it did not contain a menu as such, however participants reacted differently: one of the expert participants was aware that the page did not contain it. "It has one fault, which is the menu, I want a menu on the page. Yes, it tells me a few starters, a few seconds and such, and that's fine, but the extended menu" (P02-W1). In contrast, some non-expert participants did not find the expected menu and were not sure if it was because of 'their fault' or due to issues with the website. "the thing is that I have not been able to find, the main goal I have not achieved, eh? then, probably other blind colleagues would have found it, because I'm sure that the average of the technical resources is higher than mine" (P10-W1).

When participants were confused about whether it was because of something related to the website, the screen reader, or due to they had done something wrong. This uncertainty about not knowing what happened and why can affect the perceived Web accessibility and UX. In the W3, P05 accessed the Youtube link thinking that he would probably find information on the menu: "The thing is that I don't know if there was something else ... clearly I'm not sure that I could reach everywhere...that's why I got into Youtube, because there they might tell you" (P05-W3). "I don't know, I got lost with the buttons ... the thing is that I don't know why buttons exist ... I don't have the concepts clear about the difference between a link and a button" (P10-W4). The number of comments about how confusing Web content was completely unbalanced between 
the most accessible websites (W1, W2 get 13 comments) and the least accessible ones (W3, W4), which get 39 comments. This supports the relationship between accessibility problems and confusing situations found elsewhere (Vigo and Harper, 2013).

\subsubsection{Confusion Related to Confidence and Expertise (7)}

The uncertainty of participants was closely related to their perception of their own skills and their confidence. Intermediate and advanced participants exhibited a lack of confidence and blamed on themselves when encountering problems : "I see that I am very clumsy. If I had a better opinion about myself, I'll probably be more critical with the Web accessibility" (P10-W1). Those participants at both ends of the spectrum (i.e. beginners and experts) attributed the cause of problems to the website or the screen reader rather than to themselves.

\subsection{Experienced Web Accessibility}

After completing the tasks, we collected accessibility ratings as the websites and enquired participants about the problems they encountered. Table 2 shows the accessibility ratings given by participants on a 7-item Likert-scale where 1 indicated 'very inaccessible' and 7 'very accessible'. The median values suggest that participants perceived W1, W2 and W4 to be similarly accessible. However, a smaller standard deviation in W2 and W3 indicates a broader consensus among participants than in W1 and W4. In the case of the latter two, although opinions varied, most participants considered them to be accessible.

Table 2: Participants' experienced Web accessibility ratings (1: very inaccessible-7: very accessible)

\begin{tabular}{ccccc}
\hline website & AA compliance & median & mode & SD \\
\hline W1 & $73 \%$ & 6 & 7 & 1.95 \\
W2 & $69 \%$ & 6 & 6 & 1.42 \\
W3 & $52 \%$ & 2 & 1 & 1.62 \\
W4 & $36 \%$ & 6 & 6 & 1.95 \\
\hline
\end{tabular}

Website 1. P06 withdrew from the website as JAWS was getting blocked: "sometimes JAWS gets stuck and doesn't respond" (P06). P09 and P10 were not very confident talking about accessibility barriers: they both agreed on the large amount of visual information that W1 contained. Even if images had an alternative text, the fact that the text was not meaningful for them was perceived as a barrier: "I may have preferred a description of the person, some biographical data, or a phrase, or how the person entitles his dishes...[but] when I hear 'the portrait of our people' [as alternative text] ... this leaves me overwhelmed" (P10).

Participants also complained about not being able to find the menu even if this website did not contain a menu as such: "I haven't found any practical information, not even the menu ... you cannot access the content. Even if the sections are marked up, you click on those links and once you are in a section there is nothing there ... the most frustrating thing is going to a restaurant and not knowing what to eat" (P09). 
Website 2. Only $\mathrm{P} 01$ rated it as non-accessible, which was mainly due to network connection issues. She said the page seemed to be accessible, but because of problem when loading Web pages she could not give it a better score: "I think it's accessible, but I couldn't go beyond the links" (P01).

Website 3. All participants except P11, who said the Web page was too simple and lacked major difficulties, perceived W3 to be non-accessible. The fact that P11 did not navigate beyond the homepage may explain this outlying perception. Most participants mentioned that the navigation menu of the website was completely inaccessible because each menu item was a graphic link with the same alternative text (i.e. image). Consequently many of them did not even try to click on those links and their navigation was exclusive to the homepage.

The mental map of some participants was mismatched to the information architecture of W3. P03, who was able to distinguish between the structure from the content of the website, was ambivalent about the accessibility/nonaccessibility of the website and did not lean towards any end of the scale. According to P03, W3 had a very simple structure, which made navigation easy. However, the scarcity of content was considered a problem: "when it comes to move, you can move without any problem, the problem is related to the information in those links" (P03).

Website 4. Most participants thought W4 was quite accessible while two participants (P01 and P07) were ambivalent about it. We observed the majority of participants were not aware about the presence of non-accessible dynamic content and therefore did not perceive the accessibility barriers. Nevertheless they were suspicious about something going on: "unless I missed something to browse ...there wasn't anything or I was not able to seek...I wonder if there was a longer menu" (P09). In line with this P10 was not sure whether they were missing some content. Both P09 and P10 were disappointed and regretted their ratings when they were told that there was actually more content: "this devalues the accessibility score I gave...it's like there is a walk adapted for people with disabilities (i.e. like a safe path) and there is a hole in the middle, and I walk with my guide dog and step near the edge of the hole. I do not fall over but it could have happened" (P10).

In some cases that participants experienced a website as accessible we found comments about possibly visiting again the website, and in some cases participants commented their willingness to go to the restaurant: "I would love to go to the restaurant" (P05-W1). In contrast, in other cases when a website was not perceived to be accessible, some participants expressed their disappointment in terms of not having the intention to return to the website, neither to visit the restaurant: "if their page is not accessible, I'm not interested in the restaurant, I'd rather go to the neighbouring restaurant where I'm treated better" (P06-W3). 


\section{Discussion}

Results corroborate that blind users' experienced Web accessibility does not always correspond to compliance with accessibility guidelines. In order to better understand the experienced accessibility, we identify how experiential aspects may influence on the users' judgement about the accessibility on websites.

\subsection{Prejudices, Past Experiences and Memories Determine Expectations}

Before visiting a website most participants already had preconceived ideas and expectations regarding the website, which were mainly about on previous experiences either because of having navigated on similar websites, or due to past experiences at restaurants. For instance, three participants who had experienced uncomfortable situations when ordering at restaurants expected the website to provide them with practical information to avoid such problematic situations. Taking measures to avoid awkward situations is an strategy documented elsewhere (Ray and Ryder, 2003), which indicates that the Web is instrumental for people with disabilities to plan their journeys well in advance.

We also found that having had an endearing and satisfying experience at a particular restaurant in the past could also influence the expectations about the website of the restaurant. Nevertheless, the actual Web experience can also determine real life experiences: some participants showed either willingness to revisit the website and go to the restaurant because their good experience on the restaurant website. On the other hand, they showed no interest on restaurants and their websites if the experience was poor. This provides additional evidence to support a business case for accessibility and user experience.

Preconceptions did not only create expectations, but affected how accessibility was experienced. Participants were more demanding with those restaurants that were better positioned to have an accessible website due to their international prestige, and alleged resources and affluence. In contrast, evoked memories have an impact on the experienced Web accessibility as participants rated the accessibility of those websites that evoked fond memories higher. We suspect that traumatic memories will negatively influence too.

\subsection{Unmet Expectations}

While trying to accomplish their tasks participants' expectations are not always met. In general, this happens when there is discrepancy between what was expected and what is encountered. The underlying reasons of the mismatch are due to (i) the obstacles within the website including accessibility barriers, usability flaws and confusing information architecture; (ii) problems with the screen reader; (iii) their preferences, previous knowledge, skills and expertise.

Unmet expectations are not always explicitly reported as such: when participants come across with unexpected content or functionalities this is often reported as a problem of the website when actually there is a mismatch between the mental model of users and the model of the website. The existence of accessibility barriers made some content non reachable and therefore nonaccessible in our study. Not being able to find such content is not blamed on 
accessibility barriers which are not directly perceived, but users think that the website does not contain such content or that they were not able to find it. Specifically, intermediate and advanced users take on the responsibility of the failure, while those on the extremes (i.e. experts and beginners) blame external factors such as the website and the screen reader. In the case of beginners they may know fewer things (e.g. basic screen reader commands for Web navigation) but well learned, while experts may well know the causes of problems. When it comes to intermediate and advanced participants, they may be aware of the wide range of possibilities offered by the Web but at the same time they know the limits of their own internet skills, which lessens their confidence on their judgements.

\subsection{The Role of Uncertainty and Confidence}

Non-accessible sites create more uncertain situations than the accessible ones, which suggests there is a relationship between the the lack of accessibility and uncertainty. This finding reinforces the fact that uncertain situations trigger coping strategies (Vigo and Harper, 2013) and the fact that uncertainty is caused by unmet expectations and accessibility barriers.

While uncertain situations can be triggered when websites are visited for the first time, we observed that this uncertainty remained after finishing the task. This lasting effect has an impact beyond the perceived accessibility and affects the user experience. Therefore reducing the uncertainty would probably lead to a better user experience. Designing with familiarity and learnability principles helps in removing this uncertainty while may help boosting the experience of users (Vigo and Harper, 2014). Understandably, supporting and training users in order to acquire skills will increase their confidence to cope better with uncertainty.

\section{4. 'Accessible' Versions of Websites}

Most non-expert participants stated that they usually visit the accessible version of a website if this is available, whereas expert participants reported that they usually access the main version, even on the first time. Some participants do not access accessible versions of websites they visit periodically because alternate versions are usually inconsistent, lack information and are not updated as regularly as the main version. Some rejected accessible versions on the grounds of discrimination. Anecdotally, one participant reported that the choice depends on the navigation modality, whether navigation is exploratory or directed. On directed navigation - i.e. the user has a specific goal in mind - the accessible version is more useful. Conversely, if the navigation is of exploratory nature the main version is preferred.

'Accessible' versions do not only arise the curiosity of participants, but creates high expectations about their accessibility. Those webmasters managing different website versions must be careful because if these high expectations are not met, it generates sheer frustration. The above implies that accessible 
versions of websites should be carefully provided: they should be not only accessible, but easily findable and noticeable. For example they should be included in the results of search engines somehow along with the main website. In addition, if an accessible version exists, the link to access to it should always be easy to find.

These findings have implications on user testing practices in that the isolated aspects influence the assessment and ratings of users in a considerable way. This phenomena calls for identifying these situations and calibrating assessments and ratings on users tests.

We claim that all the above-mentioned aspects -i.e. past experiences, prejudices, evoked memories, unmet expectations and confidence - strongly affect how users perceive and experience the accessibility of websites. Because of this, we state that experienced accessibility is a highly subjective quality, which does not necessarily correspond to compliance with accessibility standards. Because guidelines are defined for standardisation purposes, their technical specification has, understandably, a unified and integral view of accessibility that can difficulty cope with the subjective aspects we have discovered.

\subsection{Methodological Considerations}

Restaurant websites were the object of this study. Participants were familiar with the product (i.e. the restaurant) but not with their website - they all were first time visitors. Following the previously mentioned classification for websites (De Marsico and Levialdi, 2004), the findings are generalisable to those websites that fall into the axes of commercial sites which target a general audience and exhibit an informative-seductive communication style. For instance, the websites of products/services such as local shops or charities that appeal to emotions in order to engage the customers.

While the outcomes are applied to blind users, we hypothesise that the results are generalisable to broader audiences. The role that prejudices, expectations and memories play on the user experience may well be independent of the users' abilities. Thus, the outcomes related to these aspects are the ones that are more directly transferable. The uncertain situations and the design barriers may also impact on able-bodied users, especially on those who are less skilled and knowledgeable about Web conventions. However, more evidence is required in order to suggest the generalisability of these outcomes to broader populations.

\section{Conclusions}

We identify the experiential aspects that influence blind users' perception and experience of accessibility barriers on the Web including

- prejudices;

- past experiences; 
- memories;

- expectations;

- and confidence;

Experienced accessibility is not only an intrinsic quality of websites, but it is also constructed from what individuals preconceive and expect from them. Consequently, these factors introduce subjectivity to the results obtained in controlled user testing protocols. The identification of the factors and calibration of results in order to correct theses deviations is critical if we want to achieve reproducible, comparable and reliable results.

\section{Acknowledgements}

We are grateful to Encarnacion Briñas for her crucial help with recruitment, and for providing facilities at the ONCE delegation; and to all participants, for their willingness to take part and contribute to the study. A copy of the websites and the results of their corresponding Web accessibility evaluations can be found in http://sipt07.si.ehu.es/amaia/stimuli. A. Aizpurua held a PhD scholarship from the Department of Education, Universities and Research of the Basque Government when the study was conducted. The work has been partly funded by the Department of Education, Universities and Research of the Basque Government (grant IT395-10) and the Basque Advanced Informatics Laboratory (BAILab), which belongs to the University of the Basque Country UPV/EHU (grant UFI11/45).

\section{References}

Abascal, J., Arrue, M., Fajardo, I., Garay, N., and Tomás, J. (2004). The use of guidelines to automatically verify web accessibility. Universal Access in the Information Society, 3(1):71-79.

Adams, A., Lunt, P., and Cairns, P. (2008). A qualititative approach to HCI research. pages $138-157$.

Aizpurua, A., Arrue, M., and Vigo, M. (2013). Uncovering the role of expectations on perceived web accessibility. In Proc. of, ASSETS, pages 74:1-74:2, New York, NY, USA. ACM.

Bargas-Avila, J. A. and Hornbæk, K. (2011). Old wine in new bottles or novel challenges: A critical analysis of empirical studies of user experience. In Proc. of, CHI, pages 2689-2698, New York, NY, USA. ACM.

Brajnik, G. (2006). Web accessibility testing: When the method is the culprit. In ICCHP, LNCS 4061, pages 156-163. Springer Verlag.

Braun, V. and Clarke, V. (2006). Using thematic analysis in psychology. Qualitative Research in Psychology, 3(2):77-101. 
Caldwell, B., Cooper, M., Reid, L. G., and Vanderheiden, G. (2008). Web content accessibility guidelines (WCAG) 2.0. http://www.w3.org/TR/WCAG20/.

Chisholm, W., Vanderheiden, G., and Jacobs, I. (1999). Web content accessibility guidelines (WCAG) 1.0. http://www.w3.org/TR/WAIWEBCONTENT/.

De Marsico, M. and Levialdi, S. (2004). Evaluating web sites: Exploiting user's expectations. International Journal of Human-Computer Studies, 60(3):381416.

Gay, G. and Li, C. Q. (2010). Achecker: Open, interactive, customizable, web accessibility checking. In Proc. of, W4A, pages 23:1-23:2, New York, NY, USA. ACM.

ISO 9241-11 (1998). ISO 9241-11: Ergonomic requirements for office work with visual display terminals (vdts) - part 11 guidance on usability.

Lazar, J., Dudley-Sponaugle, A., and Greenidge, K.-D. (2004). Improving web accessibility: a study of webmaster perceptions. Computers in Human Behavior, 20(2):269 - 288 .

McCarthy, J. and Wright, P. (2004). Technology as Experience. The MIT Press.

Petrie, H., Hamilton, F., and King, N. (2004). Tension, what tension?: Website accessibility and visual design. In Proc. of, W4A, pages 13-18, New York, NY, USA. ACM.

Power, C., Freire, A., Petrie, H., and Swallow, D. (2012). Guidelines are only half of the story: Accessibility problems encountered by blind users on the web. In Proc. of, CHI, pages 433-442, New York, NY, USA. ACM.

Ray, N. M. and Ryder, M. E. (2003). "ebilities" tourism: an exploratory discussion of the travel needs and motivations of the mobility-disabled. Tourism Management, 24(1):57 - 72 .

Rømen, D. and Svanæs, D. (2012). Validating wcag versions 1.0 and 2.0 through usability testing with disabled users. Universal Access in the Information Society, 11(4):375-385.

Sears, A. and Hanson, V. (2011). Representing users in accessibility research. In Proc. of, CHI, pages 2235-2238, New York, NY, USA. ACM.

van der Geest, T., van der Meij, H., and van Puffelen, C. (2014). Self-assessed and actual internet skills of people with visual impairments. Universal Access in the Information Society, 13(2):161-174.

Vigo, M., Arrue, M., Brajnik, G., Lomuscio, R., and Abascal, J. (2007). Quantitative metrics for measuring web accessibility. In Proc. of, W4A '07, pages 99-107, New York, NY, USA. ACM. 
Vigo, M. and Harper, S. (2013). Coping tactics employed by visually disabled users on the web. International Journal of Human-Computer Studies, 71(11):1013 - 1025 .

Vigo, M. and Harper, S. (2014). A snapshot of the first encounters of visually disabled users with the web. Computers in Human Behavior, 34(0):203 - 212. 